\title{
Intervención psicológica en trastornos del sueño: una revisión actualizada
}

\section{Psychological Intervention in Sleep Disorders: An Updated Review}

\author{
Horacio Balam Álvarez García y Ulises Jiménez Correa \\ Clínica de Trastornos de Sueño. Universidad Nacional Autónoma de México
}

\begin{abstract}
Resumen: Los trastornos de sueño son una problemática recurrente en la actualidad. A pesar de los múltiples estudios sobre intervenciones eficaces, es poca su difusión en la literatura hispanohablante. A partir de esta necesidad, el objetivo del presente artículo es dar a conocer las intervenciones con mayor efectividad sobre los trastornos del sueño, como son el Insomnio, Síndrome de Apnea-Hipopnea Obstructiva de Sueño (SAHOS) y Narcolepsia. Se revisó: características del sueño, criterios diagnósticos con base en la clasificación internacional de trastornos de sueño y las intervenciones psicológicas para cada uno los trastornos antes mencionados.
\end{abstract}

Palabras clave: insomnio, SAHOS, narcolepsia, Terapia Cognitivo Conductual

\begin{abstract}
Sleep disorders are a recurring problem today. In spite of multiple studies on effective interventions, there is little diffusion in Spanish-speaking literature. From this need, the objective of this article is to present the most effective interventions on sleep disorders, such as Insomnia, Obstructive Sleep Apnea-Hypopnea Syndrome (OSAH) and Narcolepsy. We reviewed: sleep characteristics, diagnostic criteria based on the international classification of sleep disorders, and psychological interventions for each of the aforementioned disorders. Keywords: insomnia, OSAH, narcolepsy, Cognitive Behavioral Therapy
\end{abstract}

El sueño tiene un papel importante en la vida del ser humano y durante siglos se han dado diversas explicaciones sobre sus características y funciones. En un inicio, la explicación se centraba en la función que tenían los ensueños en la vida del hombre vinculándolos con aspectos de índole religioso, siendo utilizado como una forma de comunicarnos con los dioses, es así, que la utilidad del sueño estaba dirigida a predecir y entender el mundo que nos rodea (Hoffman, 2004).

Horacio Balam alumno de nivel maestría del Dr. Ulises Jímenz en la Clínica de Trastornos de Sueño.

Ulises Jiménez es docente de la Universidad Nacional Autónoma de México.

La correspondencia sobre este artículo debe ser enviada a Horacio Balam al e-mail: hbgarcia mosh11@hotmail.com

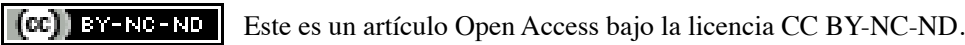


Con el paso de los siglos, los científicos se fueron alejando más de esta visión religiosa y comenzaron a realizar estudios del sueño bajo una visión totalmente distinta. Los estudios realizados por Hans Berger con el electroencefalograma (EEG) permitieron registrar la actividad eléctrica cerebral del ser humano. Berger reportó que durante la vigilia se observaba actividad eléctrica rápida y de bajo voltaje mientras que durante el sueño observó actividad eléctrica lenta y de alto voltaje (Berger, 1929).

Basándose en los cambios de actividad eléctrica en el EEG se realizó una primera clasificación de los estados de sueño en el ser humano (Loomis et al., 1937). Los estudios de Aserinsky y Kleitman (1953) marcaron un cambio al reportar que los estados de sueño se interrumpían por periodos de actividad eléctrica rápida parecidos a los observados en la vigilia y movimientos oculares rápidos, lo que se conoce actualmente como sueño MOR. El estudio de sueño MOR llevó a reportar que durante el mismo se producían los ensueños (Dement y Kleitman, 1957). Los estudios posteriores de las diversas fases de sueño han dado como resultado teorías sobre los mecanismos fisiológicos relacionados con el sueño, las alteraciones que se presentan durante el sueño y finalmente las consecuencias de una inadecuada calidad de sueño (Thorpy, 1997).

La psicología ha auxiliado a la investigación del sueño, brindando teorías sobre la función que tiene el sueño en el ser humano. El primer acercamiento fue propuesto por Sigmund Freud a través de la función que tiene el sueño en el inconsciente, el cual se apoyó en el análisis e interpretación de los sueños para determinar el origen de las afectaciones mentales (Freud, 1923).

Actualmente existen aproximaciones psicológicas en el marco de las ciencias cognitivas sobre el sueño, como es en la consolidación de diversas funciones cognoscitivas (Wilhem et al., 2011) y el impacto en la salud ante las alteraciones de la misma (Van Someren et al., 2015).

\section{El sueño y sus características}

De acuerdo a la División de Medicina de Sueño de la Escuela de medicina de Harvard: "el sueño es un estado que se caracteriza por cambios en la actividad de las ondas cerebrales, la respiración, la frecuencia cardíaca, la temperatura corporal y otras funciones fisiológicas" (School y Foundation, n.d.).

Partiendo de esta definición podemos dividir el sueño en diferentes fases: a) Vigilia: estado inicial del funcionamiento en el que existe actividad beta, de bajo voltaje, con tono muscular y actividad ocular: b) Sueño sin movimientos oculares rápidos que comprende tres etapas: Etapa 1, la actividad theta se retarda, disminuyen los movimientos oculares en comparación con la vigilia y la actividad muscular disminuye. Etapa 2, aparecen periodos de actividad theta rápida, conocidos como husos de sueño, así como complejos K que son espigas de alto voltaje. Etapa 3, existe frecuencia de actividad theta en un inicio para después cambiar a una frecuencia de actividad delta. Al igual que las etapas anteriores, no hay movimiento de los ojos y la actividad muscular está disminuida.; c) Sueño de movimientos oculares rápidos (MOR), existe una actividad theta rápida y de baja frecuencia; no hay actividad muscular y los ojos tienen movimientos rápidos, durante mucho tiempo se ha observado que en esta fase se producen mayoritariamente los ensueños, aunque actualmente se reporta que se pueden presentar en menor medida en fases del sueño sin MOR ( Cipolli, et al., 2016; Lombardo-Aburto, 2011; Soriano Mas, 2007).

A partir de los estudios de estas fases, se comenzó a discutir la función de los sueños. Algunas investigaciones determinaron que el sueño no se puede tomar como una fase de inactividad; si bien no existe la actividad física, los componentes electrofisiológicos demostraron que existe una elevada actividad mental (Medrano-Martínez y Ramos-Platón, 2014).

Estudios de neuroimagen han reportado actividad en la corteza visual de asociación y en la corteza pre frontal, esta última participa en planificar la conducta, mantenerse al tanto de cómo se organiza la secuencia temporal de acontecimientos y distinguir entre realidad e ilusión (Carlson, 2014). También se ha reportado la relevancia del sueño en la consolidación de la memoria, el aprendizaje y la depuración de información poco relevante almacenada durante el día (Tirapu-Ustárroz, 2012). Además de su relación con cambios en el sistema cardiovascular, sistema respiratorio, sistema endocrino, sistema inmune y temperatura corporal (Contreras, 2013). 
Por ello, cuando se detecta alguna alteración dentro del ciclo de sueño, se determina que existe un trastorno del sueño. Con ello, es importante brindar un diagnóstico certero sobre dicha alteración y así orientar un adecuado tratamiento.

\section{Trastornos del sueño}

De acuerdo con la Clasificación Internacional de Trastornos del Sueño, en su tercera edición (ICSD -3) de la Academia Americana de Medicina de Sueño (AASM) los trastornos del sueño: "son una alteración en el ciclo de sueño - vigilia, en el que se ven afectados la consolidación y mantenimiento del sueño repercutiendo en distintas áreas de funcionamiento de la persona" (American Academy Sleep Medicine, 2014).

Con base en el ICSD-3, encontramos que las alteraciones de sueño se dividen en seis categorías clínicas: 1) Insomnio, 2) Trastornos respiratorios del sueño, 3) Trastornos centrales de hipersomnolencia, 4) Trastornos del ritmo circadiano del sueño-vigilia, 5) Parasomnias y 6) Trastornos del movimiento durante el sueño (American Academy Sleep Medicine, 2014).

A nivel mundial, se reporta que un $45 \%$ de la población padecen algún tipo de trastorno de sueño. En los Estados Unidos de América (EUA) la prevalencia de estos padecimientos oscila entre un $10 \%$ y un $30 \%$ en adultos de entre 30 y 64 años, mientras que en los adultos mayores el problema presenta hasta en un $50 \%$. Encontrando que la apnea obstructiva de sueño es el trastorno con mayor prevalencia (4.2\%), seguido por insomnio $(1.2 \%)$, el movimiento periódico de extremidades $(0.4 \%)$ y en casos menores narcolepsia $(0.04 \%)$ (Ohayon, 2011; Ram et al., 2010).

Los tratamientos para trastornos del sueño se enfocan en las características propias del trastorno, que van desde intervenciones quirúrgicas, tratamiento farmacológico, accesorios complementarios para el dormir y tratamiento psicológicos con base empírica, estos últimos son un campo relativamente nuevo (Chokrovetry, 2011; Chokroverty y Thomas, 2014; Montserrat Canal y Puertas Cuesta, 2015).

\section{Antecedentes de intervención psicológica en Insomnio}

Las primeras intervenciones no farmacológicas o psicológicas en los trastornos del sueño, se enmarcaron dentro del campo de la medicina conductual (Godoy, 1998; Reynoso-Erazo y Becerra-Galvez, 2014). Los objetivos de intervención son brindar herramientas terapéuticas para el manejo de las variables psicológicas que están presentes antes, durante o después del dormir (Cheung et al., 2018).

Las primeras propuestas de intervención se enfocaron en la reducción de hiperactivacion de la persona que padece insomnio. Siendo en los años 30`s, la relajación muscular progresiva la primera propuesta que abordó desde esta perspectiva este padecimiento (Jacobson, 1938; Millet, 1938).

En esta línea, las investigaciones subsecuentes se enfocaron a disminuir los componentes fisiológicos de los pacientes con insomnio, analizando la eficacia de la relajación a través de auto reportes de estudio de caso (Weil y Goldfried, 1973), retroalimentación biológica (Biofeedback) (Borkovec y Weerts, 1976; Borkovec et al., 1979) técnicas de relajación muscular en combinación con drogas sedantes (Borkovec y Fowles, 1973) o con otras técnicas terapéuticas como la intención paradójica (Nicassio y Bootzin, 1974; Geer y Katkin, 1966; Ascher y Turner, 1979).

A pesar de las múltiples investigaciones que demostraron la eficacia de la relajación en los componentes de hiperactivacion dentro del insomnio, las diferencias clínicas arrojaron que el componente de relajación no era el factor que desarrollaba y mantenía la problemática (Monroe, 1967).

Es a través de la propuesta de Bootzin, que el tratamiento del insomnio comienza a tener una orientación conductual, ya que el modelo explicativo de inicio y mantenimiento del padecimiento se explica por un proceso de condicionamiento clásico y se mantiene debido a un condicionamiento operante. Aunado a esta nueva conceptualización, se propone el control de estímulos (Bootzin., 1972).

Bajo este nuevo modelo de intervención, las investigaciones sobre la aplicación de técnicas combinadas de 
terapia de conducta, comenzaron a crecer demostrando que los componentes conductuales tenían una eficacia mayor que la relajación sola y tenían buen nivel de eficacia cuando se combinaban (Bootzin et al., 1991).

Sentadas las bases de la Terapia de Conducta para el Insomnio durante los años ochenta, los tratamientos tuvieron una nueva faceta al incorporar una nueva técnica conocida como restricción de tiempo en cama (actualmente se conoce como restricción de sueño). Esta técnica aumentó los componentes del tratamiento fortaleciendo la evidencia de eficacia y preparando el campo para protocolos de investigación con mayor grado de control e impacto (Bootzin y Nicassio,1978; Lacks et al., 1983; Kales y Kales, 1984).

Sin embargo, la investigación fue demostrando que los componentes fisiológicos y conductuales, eran solo una parte de los factores que mantenían el insomnio, es así que, se comenzó a tomar en cuenta la relación de los pensamientos con el malestar clínico en el insomnio (Morin, 1993; Morin et al., 1993; Morin,1994 ).

Con la integración de los componentes de pensamiento, se comenzó a observar que los mismos se interrelacionaban con los factores conductuales y los fisiológicos, creando un patrón de reforzamiento (Harvey, 2002). Con ello se comenzó a diseñar tratamientos multicomponentes que acogían cada una de las técnicas ya investigadas y se comenzó a desarrollar el tratamiento psicológico del insomnio (Harvey, 2005).

Es así que, el desarrollo del tratamiento psicológico estándar comenzó por analizar los componentes con mayor grado de eficacia (Buysse et al., 2006) para después comenzar a desarrollar protocolos de investigación controlados y con muestras aleatorizadas (Morin et al., 2009; Sivertsen et al., 2006). El resultado de estas investigaciones fue engrosando el nivel de evidencia y posicionando la terapia cognitivo conductual como tratamiento estándar en el insomnio.

\section{Insomnio}

De acuerdo a la clasificación internacional de los trastornos de sueño (ICSD-3), el Insomnio se define como: "Dificultad persistente en el inicio del sueño, su duración, consolidación o calidad que ocurre a pesar de la existencia de adecuadas circunstancias y oportunidad para el mismo y que se acompaña de un nivel significativo de malestar o deterioro de las áreas social, laboral, educativa, académica, conductual o en otras áreas importantes del funcionamiento humano" (American Academy Sleep Medicine, 2014).

Dividiéndose en:

- Trastorno del Tipo Insomnio Crónico: Se caracteriza por quejas en inicio o en el mantenimiento del sueño, síntomas de somnolencia durante el día con un mínimo de tres episodios por semana en los últimos tres meses.

- Trastorno del Tipo de Insomnio Agudo: Se caracteriza por dificultad para el inicio o el mantenimiento del sueño, que resulta en insatisfacción con el sueño y que aparece por periodos de tiempo inferiores a los tres meses. En algunos casos hay una causa identificable que puede actuar como precipitante y en otros casos ocurre episódicamente coincidiendo con factores precipitantes.

- Otros Tipos de Insomnio: Este diagnóstico se reserva para personas con síntomas de dificultad de inicio y mantenimiento del sueño que no cumplen los criterios de insomnio crónico o insomnio agudo y, por lo tanto, este diagnóstico se usa de forma provisional en espera de más información para establecer el diagnóstico definitivo (American Academy Sleep Medicine, 2014).

El tratamiento actual desde la perspectiva psicológica se denomina como Terapia Cognitivo Conductual para Insomnio (TCC-I), esto se debe a que existe evidencia que avala la eficacia de esta intervención (Trauer et al., 2015)

Para entender cómo se fundamenta esta intervención, debemos revisar brevemente el modelo de las 3P. Llamado así debido a que se analizan tres factores denominados predisponentes, precipitantes y perpetuantes. El modelo propone que el Insomnio se mantiene debido a que dentro de estos factores existen variantes conductuales y cognitivas que perpetúan el padecimiento (Perlis et al., 2011).

Los factores Predisponentes, son aspectos físicos de la persona como la segregación de neurotramisores específicos o manifestaciones genéticas que pueden generar alteraciones en el ciclo de sueño como conductas 
características de estados de ansiedad o un estado mayor de activación fisiológico (Riemann, et al., 2010; Viola, et al., 2007).

Esta predisposición se conjunta con circunstancias estresantes en la vida que pueden detonar el Insomnio, este segundo componente se le denomina como factor precipitante. Por ejemplo; la muerte de un ser querido, pérdida del empleo, etc. Pero conforme pasa el tiempo estas circunstancias desaparecen y a su vez el Insomnio (Bastien et al., 2004).

Cuando esto no sucede, se analizan las circunstancias que lo están manteniendo, esto puede ir desde actividades incompatibles con el sueño, como es ver la televisión en la cama, leer, realizar actividad física antes de acostarse, a estas circunstancias se le denomina como factores perpetuantes (Ebben y Spielman, 2009).

El objetivo del tratamiento psicológico está encaminado a: brindar una alternativa que no genere ningún tipo de consecuencia secundaria, como es la dependencia derivada del tratamiento farmacológico. Por ello, se pretende disminuir los componentes conductuales (hábitos inadecuados de sueño, horarios irregulares de sueño), cognitivos (expectativas poco realistas, preocupaciones y creencias inútiles) y psicofisiológicos (tensión física y mental e hiperactivacion) que mantienen el Insomnio (Morin y Benca, 2012).

\section{Tabla1. Técnicas de intervención en insomnio}

\section{Técnica Descripción}

Restricción del Se restringe el tiempo que pasa el paciente en la cama despierto, indicando que solo debe acostarse tiempo en cama cuando se desee dormir. Con ello se busca que el paciente recupere el impulso de sueño homeostático.

Control de Instrucciones que se brindan al paciente diseñadas para aumentar la asociación entre el dormitorio estímulos $\quad$ y la cama con el sueño, disminuyendo las actividades que promueven la vigilia.

Relajación mus- Entrenamiento que se brinda al paciente en el cual se alterna la tensión muscular con la distensión cular progresiva de diferentes pares musculares. Se busca disminuir la hiperactivacion que tiene el paciente.

Biofeedback Consiste en presentar información al paciente de forma inmediata de sus procesos fisiológicos durante la vigilia a través de equipos específicos, con esta presentación de información se busca la relajación del paciente.

Mindfulness Práctica de meditación consiente, en la que el paciente atiende sin juzgar sus estados internos prestando atención a los cambios previos al inicio de sueño, se busca con ello que el paciente se relaje.

Reestructura- Utilizando cuestionamientos socráticos, se busca que el paciente cambie sus pensamientos disfunción Cognitiva cionales hacia el no dormir y la preocupación excesiva, por pensamientos más realistas y adaptativos.

Intención Se busca que el paciente realice las conductas o pensamientos que le impiden dormir en lugar de paradójica evitarlas, con la finalidad de disminuir la preocupación asociada al no poder dormir. Por ejemplo se pide al paciente que al acostarse se mantenga despierto lo mayor posible.

Higiene de Normas que se le brindan al paciente que le ayuden a tener condiciones ambientales adecuadas sueño $\quad$ para poder tener un sueño adecuado.

Fuente: Elaboración propia de los autores. 
Dentro de las técnicas que se han propuesto en la TCC-I, las que han demostrado tener mayor evidencia son las conductuales (control de estímulos y restricción del tiempo en cama, relajación muscular progresiva), seguidas por las cognitivas (reestructuración cognitiva, intención paradójica) y finalmente las medidas de higiene de sueño (Perlis et al., 2009).

Además de las técnicas ya mencionadas se ha intentado explorar diferentes técnicas que tratan de disminuir los síntomas en el insomnio. La mayoría de ellas se centra en la relajación, que indirectamente disminuyen la hiperactivacion y vigilancia de la persona con insomnio a pesar de ello su nivel de eficacia no es tan alentador por lo que solo se llegan a utilizar como complemento a la TCC-I estándar (Chokroverty, 2011).

Los tratamientos de Biofeedback tienen el objetivo de disminuir la hiperactivacion del paciente insomne dando parámetros objetivos de la misma sobre el nivel de activación, los niveles de alerta y de actividad límbica. A pesar de los avances en esta área, existen resultados controvertidos sobre la eficacia y se ha determinado que puede ser usado como complemento de la TCC-I (Lovato et al., 2018).

La técnica de Mindfulness o atención plena propuesta por John Kabat Zinn (Kabat-Zinn, 2007) ha sido utilizada como una técnica de relajación entre los pacientes insomnes, aunque los resultados obtenidos son menores o iguales a las técnicas de relajación convencionales (Cheong et al., 2018).

A pesar de los resultados alentadores de la misma, se ha observado que Mindfulness no muestra mayor eficacia que las técnicas de relajación convencional y no es mayor al efecto placebo. A pesar de ello se ha dado propuestas básicas de trabajo desde esta perspectiva y se han desarrollado investigaciones pertinentes (ver tabla 1) (Rash et al., 2019).

Además de las técnicas anteriormente mencionadas, se han generado programas de trabajo basado en acupuntura que tienen como objetivo bloquear los puntos psicofisiológicos de activación presentes en el insomnio. Actualmente estas técnicas no tiene suficiente sustento empírico por lo que solo se recomienda el trabajo como terapia complementaria dependiendo del usuario (Cao et al., 2019).

Es así que cada una de estas técnicas se encamina en disminuir la sintomatología del Insomnio, para ello se trabaja con una estructura general de programación que se compone de 4 a 6 sesiones (una por semana preferentemente), la modalidad en la que se puede dar es a nivel individual y grupal, el objetivo final es que el paciente tenga una mejora significativa en la calidad de sueño, tiempo total de sueño y un ajuste en las áreas personales del paciente (Morin et al., 2003).

Con estos antecedentes, los componentes de la TCC-I comenzaron a adaptarse a otros trastornos de sueño debido a la comorbilidad que existe entre ellos, con la finalidad de mejorar el apego al tratamiento convencional de cada uno de ellos y mejorar la calidad de vida de los pacientes, por ello revisaremos los trastornos que han comenzado a tener adaptaciones de intervención psicológica.

\section{Síndrome de apnea-hipopnea obstructiva de sueño}

Es un trastorno caracterizado por episodios de obstrucción de vía respiratoria durante el sueño, teniendo como síntomas principales fatiga, ronquidos intensos y somnolencia diurna excesiva (SDE). Existen dos subdivisiones, de acuerdo al tipo de obstrucción, denominados hipopnea cuando la obstrucción es parcial y apnea obstructiva cuando la obstrucción es completa (Hernández Marín y Herrera, 2017).

La evaluación del Síndrome de apnea-hipopnea de sueño (SAHOS) se compone por diferentes áreas, iniciando con la exploración física en las que se revisan características en el rostro, la boca y las vías respiratorias que se relacion con la obstrucción aérea, además de analizar el peso corporal del paciente. Por otra parte se analizan los síntomas diurnos del paciente, a través de diferentes escalas de hábitos y calidad de sueño. Finalmente se realizan estudios de laboratorio como la Polisomnografia con la finalidad de observar el número de episodios durante la noche y el test de latencia múltiples de sueño que nos ayudará a determinar el nivel de somnolencia que presenta el paciente (Fong et al., 2005; Paez-Moya y Parejo-Gallardo, 2017).

Cuando se tiene los resultados y se cuenta con un diagnóstico preciso, los tratamientos para SAHOS comprenden intervenciones con aparatos específicos como mascarillas que generan una presión positiva en la vía aérea (CPAP por sus siglas en inglés) dispositivos orales, manejo quirúrgico, manejo farmacológico y recomendaciones conductuales (Chokroverty y Thomas, 2014). 
Las recomendaciones conductuales en el SAHOS se derivan de la TCC-I debido a la comorbilidad que se presenta con el insomnio presentando dificultades en el uso regular de las mascarillas para el CPAP, principalmente un bajo apego al uso o variables de ansiedad e hiperactivacion durante su uso (Luyster et al., 2010; Pich, et al., 2013; Wickwire et al., 2010).

La intervención tiene por objetivo elevar el apego al tratamiento y disminuir los síntomas de insomnio, los componentes que se retoman son: a) Psicoeducación en las que se explica el uso adecuado de la mascarilla, en que consiste, así como posturas adecuadas a la hora de dormir y las medidas de higiene de sueño para tener rutinas adecuadas para el inicio de sueño (Escobar-Cordoba y Echeverry-Chabur, 2017; Wozniak et al., 2014) b) La restricción del tiempo en cama, con la finalidad de que el paciente pase el menor tiempo posible en el dormitorio antes de iniciar la sueño (Spielman et al., 1987), c) Reestructuración Cognitiva, para la modificación de pensamientos irracionales sobre el sueño (Belanger et al., 2006).

Se ha reportado que esta modalidad de intervención, ha mostrado eficacia al aumentar la adherencia al tratamiento de CPAP y al uso de la mascarilla en comparación con tratamiento habitual, también se observó una remisión en los Sintomas de insomnio a los 6 meses de seguimiento (Sweetman et al., 2019). Estos resultados concuerdan con la disminución de los resultados de la escala de somnolencia de Epworth de pacientes con SAHOS previamente reportados (Sweetman et al., 2017).

\section{Narcolepsia}

Representa uno de los trastornos de sueño con menor índice de prevalencia, reportándose a nivel mundial un caso por cada 2,000 habitantes. Dentro de las principales características se encuentran alteraciones en el sistema hipocretinérgico, lo que muestra una disminución de hipocretinas en el líquido céfalo raquideo. De acuerdo con la ICDS-3, la narcolepsia se clasifica en tipo 1 y tipo 2 según el nivel de hipocretinas (Goswami et al., 2010; Overeem et al., 2012). Anteriormente se creó la hipótesis de que existía una prevalencia genética, pero al encontrar datos del $25 \%$ de incidencia en gemelos monocigótos sugiere que hay baja heredabilidad (Mignot, 1998).

Los síntomas que caracterizan a este trastorno son la somnolencia excesiva diurna, cataplejía, alucinaciones hipnagógicas e hipnopómpicas, parálisis de sueño y un sueño nocturno fragmentado. Se reporta que los episodios de cataplejía están relacionados con episodios de emoción intensa que el paciente no logra procesar por lo que el cuerpo pierde el tono muscular (de forma parcial o total), aunado a ello la SDE repercute en el funcionamiento general del paciente, afectado las áreas laborales y familiar, impactando en la calidad de vida (Goswami, 2012; Pabon, et al., 2010).

La intervención de primera línea es la farmacoterapia, utilizando principalmente estimulantes, oxibato de sodio y antidepresivos que tienen como objetivo la estabilización de los síntomas como SDE, cataplejía y sueño fragmentado. El tratamiento es de por vida y se deben realizar ajustes constantes (Kornum et al., 2017; Majid y Hirshkowitz, 2010).

En apoyo al tratamiento farmacológico existen propuestas generales de intervención basadas en cambios de comportamiento como son: entrenamiento en hábitos de sueño y orientación psicosocial que complementan pero no han mostrado uniformidad en el uso clínico (Bassetti, et al., 2019). Otro de los manejos conductuales que han mostrado eficacia, es la programación de siestas (Mullington y Broughton, 1993). Esta técnica tiene por objetivo mejorar el estado de alerta y disminuir la SDE, los resultados obtenidos muestran un aumento en la latencia de sueño aunque no se observaron diminución en los ataques de sueño (Rogers y Aldric, 1993).

A partir de las limitaciones observadas se ha propuesto un modelo cognitivo-conductual de la narcolepsia, en la que encontramos tres principios explicativos. El primero nos habla de una interacción constante de factores de riesgo externo (ejemplo: situaciones emocionales intensas) y factores de riesgo interno (ejemplo: alteraciones neuroquímicas como lo son las hipocretinas), que se encuentran en una relación covariada de tres procesos denominados predisponentes (ejemplo predisposición genética) precipitantes (aspectos físicos del paciente) y desencadenantes (circunstancias relacionadas con la narcolepsia pero que por sí sola no la explica, por ejemplo las creencias irracionales). El segundo principio nos habla de dimensiones interactivas relacionadas con 
condiciones ambientales de respuesta. El tercer, y último, principio es la interacción entre los subprocesos y la retroalimentación de todo el sistema (Marín-Agudelo y Vinaccia, 2005).

Bajo este modelo, la intervención psicológica estaría dirigida a utilizar estrategias de afrontamiento que disminuyan la SDE, se afrontan las creencias irracionales sobre los episodios de cataplejía y se promueva un sueño de mayor calidad para el paciente. Dentro de las estrategias encontramos medidas de higiene de sueño, terapia de control de estímulos, técnicas de relajación, terapia cognitiva y la creación de horarios con siestas programadas (Marín-Agudelo y Vinaccia, 2005).

\section{Discusión y conclusiones}

El grueso de la literatura pone de manifiesto que la Terapia Cognitivo Conductual es un tratamiento válido para el manejo del insomnio (Geiger-Brown et al., 2015; Traueret al., 2015) derivado de ello se han comenzado a mejorar los protocolos, obteniendo formatos abreviados de 4 sesiones (Pigeon et al., 2017; Tanaka et al., 2019) y manejo basado en internet, (Luyster et al., 2020; Moloney et al., 2019;) obteniendo resultados similares a los tratamientos convencionales lo que ayuda a la disminución en el costo de la intervención.

Lo componentes utilizados en el insomnio se han comenzado a utilizar en trastornos comórbidos como el SAHOS. Actualmente la intervención psicológica en SAHOS se ha centrado en disminuir los síntomas de insomnio e incrementar la adherencia terapéutica de los pacientes, obteniendo resultados prometedores (Sweetman et al., 2020).

A pesar de ello, las características de los trastornos de sueño dificultan la adecuación de la intervención, como lo es en la narcolepsia, ya que, aunque existe un modelo explicativo la baja prevalencia ha llevado a que el abordaje sea multivariado y que el objetivo sea encaminado a sólo uno de los síntomas y no en su totalidad. Actualmente se dispone de una propuesta de intervención basada en el modelo cognitivo conductual de narcolepsia, aunque aún no se ha reportado algún tipo de estudio donde se le implemente (Marín Agudelo et al., 2014).

Como podemos observar el campo de los trastornos de sueño ha tenido un amplio desarrollo en disciplinas médicas, lo que consolido una subdiciplina llamada medicina de sueño, este campo multidisciplinar se ha mostrado abierto a las intervenciones psicológicas con miras de reducir costos de tratamiento y mejorar el impacto en los pacientes, para ello se ha generado un área denominada medicina conductual de sueño en la cual englobamos las intervenciones descritas (Espie, 2013; Stepanski, 2003).

Finalmente es importante mencionar que la intervención psicológica en este campo debe ser llevada a cabo por profesionales con formación en las áreas de sueño y de terapia cognitivo conductual, para garantizar el adecuado manejo del paciente y la detección oportuna de la patología, teniendo como resultado una mejor calidad de vida.

\section{Referencias}

American Academy Sleep Medicine. (2014). International Clasification of Sleep Disorders -ICSD-3 ( $3^{\mathrm{a}}$ ed.). Autor.

Ascher, L. y Turner, R. (1979). Paradoxical intention and insomnia: An experimental investigation. Behaviour Research and Therapy, 17(4), 408-411. https://doi.org/10.1016/0005-7967(79)90015-9

Aserinsky, E. y Kleitman, N. (1953). Regularly ocurring periods of eye motility and concomitant phenomena during sleep. Science, 118(3062), 273-274. https://doi.org/10.1126/science.118.3062.273

Bassetti, C., Adamantidis, A., Burdakov, D., Han, F., Gay, S., Kallweit, U., Khatami, R., Koning, F., Kornum, B. R., Lammers,G. J., Liblau, R. S., Luppi, P. H., Mayer, G., Pollmächer, T., Sakurai, T., Sallusto, F., Scammell,T.E., Tafti, M. y Dauvilliers, Y. (2019). Narcolepsy - clinical spectrum, aetiopathophysiology, diagnosis and treatment. Nature Reviews Neurology, 15(9), 519-539. https://doi.org/10.1038/s41582-019-0226-9.

Bastien, C., Vallieres, A. y Morin, C. (2004). Precipitating factors of insomnia. Behavioral Sleep Medicine, 2(1), 50-62. https://doi.org/10.1207/s15402010bsm0201 5

Belanger, L., Savard, J. y Morin, C. (2006). Clinical management of insomnia using cognitive therapy. Behavioral Sleep Medicine, 4(3), 179-202. https://doi.org/10.1207/s15402010bsm04034 
Berger,H.(1929). Über das elektroencephalogramm des Menschen. Archiv für Psychiatrie und Nervenkrankheiten, 87, 527-570. https://doi.org/10.1007/BF01797193

Bootzin, R. (1972). Stimulus control treatment for Insomnia. Proceedings of the 80th Annual Convention of the American Psychological Association, 7, 395-396.

Bootzin, R. y Nicassio, P. (1978). Behavioral treatments for insomnia. En M. Hersen, R. Eisler y P. Miller, Progress in behavior modification (pp. 1-47). Academic Press.

Bootzin, R., Epstein, D. y Wood, J. (1991). Stimulus control instructions. En P. Hauri, Case studies in insomnia. (pp. 19-28). Plenum Medical.

Borkovec, T. y Fowles, D. (1973). Controlled investigation of the effects of progressive and hypnotic relaxation on insomnia. Journal of Abnormal Psychology, 82(1), 153-158. https://doi.org/10.1037/h0034970

Borkovec, T. y Weerts, T. (1976). Effects of progressive relaxation on sleep disturbance An electroencephalographic evaluation. Psychosomatic Medicine, 38(3), 173-180. https://doi.org/10.1097/00006842197605000-00003

Borkovec, T., Grayson, J., O'Brien, G. y Weerts, T. (1979). Relaxation treatment of pseudoinsomnia and idiopathic insomnia: An electroencephalographic evaluation. Journal of Applied Behavior Analysis, , 12(1), 3754. https://doi.org/10.1901/jaba.1979.12-37

Buysse, D., Ancoli-Israel, S., Edinger, J., Lichstein, K. y Morin, C. (2006). Recommendations for a standard. Sleep, 29(9), 1155-1173. https://doi.org/10.1093/sleep/29.9.1155.

Cao, H., Yu, M., Wang, L., Fei, Y., Xu, H., y Liu, J. (2019). Acupuncture for Primary Insomnia: An Updated Systematic Review of Randomized Controlled Trials. The Journal of Alternative and Complementary Medicine, 25(5), 451-474. https://doi.org/10.1089/acm.2018.0046

Carlson, N. (2014). Fisiología de la conductua (11 ${ }^{\mathrm{a}}$ ed.). Pearson Educacion.

Cheong, M., Lee, G., Kang, H., Kim, S., Kim, H., Jo, H., Kang, Y., Kim, J. y Baek, H. (2018). Clinical effects of mindfulness meditation and cognitive behavioral therapy standardized for insomnia: A protocol for a systematic review and meta-analysis. Medicine (Baltimore), 97(51), Artículo e13499. https://doi.org/10.1097/ MD.0000000000013499

Cheung, J., Jarrin, D., Ballot, O., Bharwani, A. y Morin, C. (2018). A Systematic Review of Cognitive Behavioral Therapy for Insomnia Implemented in Primary Care and Community Settings. Sleep Medicine Reviews. 44,23-36. https://doi.org/10.1016/j.smrv.2018.11.001

Chokroverty, S. (2011). Medicina de los trastornos del sueño. Aspectos básicos, consideraciones técnicas y aplicaciones clínicas. Elsevier España.

Chokroverty, S. y Thomas, R. (2014). Atlas of Sleep Medicine. Elsevier Inc.

Cipolli, C., Ferrara, M., De Gennaro, L. y Plazzi, G. (2016). Beyond the neuropsychology of dreaming: insights into the neural basis of dreaming with new techniques of sleep recording and analysis. Sleep Medicine Reviews, 35, 8-20. https://doi.org/10.1016/j.smrv.2016.07.005

Contreras, S. (2013). Sueño a lo largo de la vida y sus implicaciones en salud. Revista Médica Clínica Las Condes, 24(3), 341-349. https://doi.org/10.1016/S0716-8640(13)70171-8

Dement, W. y Kleitman, N. (1957). The relation of eye movement during sleep to dream activity. Journal of Experimental Psychology, 53(5), 339-346. https://doi.org/10.1037/h0048189

Ebben, M. y Spielman, A. (2009). Non-pharmacological treatments for insomnia. Journal Behaviour Medicine, 32(3), 244-254. https://doi.org/10.1007/s10865-008-9198-8

Escobar-Cordoba, F. y Echeverry-Chabur, J. (2017). Psicoeducación en el síndrome de apnea-hipopnea obstructiva del sueño (SAHOS). Revista Facultad de Medicina, 65(1), 105-107. https://doi.org/10.15446/revfacmed.v65n1Sup.59544

Espie, C. (2013). Behavioural sleep medicine conceptualisations and associated treatment of clinical insomnia disorder in adults. University of Oxford.

Fong, S., Ho, C. y Wing, Y. (2005). Comparing MSLT and ESS in the measurement of excessive daytime sleepiness in obstructive sleep apnoea syndrome. Journal Psychosomathic Research, 58(1), 55-60. https://doi. org/10.1016/j.jpsychores.2004.05.004

Freud, S. (1923). La interpretacion de los sueños. Iztaccihuatl. 
Geer, J. y Katkin, E. (1966). Treatment of insomnia using a variant of systematic desensitization: A case report. Journal of Abnormal Psychology, 71(3), 161-164. https://doi.org/10.1037/h0023382

Geiger-Brown, J., Rogers, V., Liu, W., Ludeman, E., Downton, K. y Diaz-Abad, M. (2015). Cognitive behavioral therapy in persons with comorbid insomnia: A meta-analysis. Sleep Medicine Reviews, 23, 54-67. https:// doi.org/10.1016/j.smrv.2014.11.007

Godoy, J. (1998). Medicina Conductual. En V. Caballo, Manual de técnicas de terapia y modificacion de conducta (pp. 871-891). Siglo veintiuno editores.

Goswami, M. (2012). Quality of life in narcolepsy. Sleep Medicine Clinical, 7(21), 341-351. https://doi. org/10.1016/j.jsmc.2012.03.006

Goswami, M., Pandi-Perumal, S. y Thorpy, M. (2010). Narcolepsy A clinical guide. Humana Press.

Harvey, A. (2002). A cognitive model of insomnia. Behaviour Research and Therapy Journal, 40(8), 869-893. https://doi.org/10.1016/S0005-7967(01)00061-4

Harvey, A. (2005). A cognitive theory of and therapy for chronic insomnia. Journal of Cognitive Psychotherapy: An International Quarterly, 19(1), 41-60.

Harvey, A. y Payne S. (2002). The management of unwanted pre-sleep thoughts in insomnia: distraction with imagery versus general distraction. Behaviour Research Therapy, 40(3), 267-277. https://doi.org/10.1016/ S0005-7967(01)00012-2

Hernandez Marin, L. y Herrera, J. (2017). Protocolo para el síndrome de apnea hipopnea obstructiva del sueño en adultos.Recomendaciones actuales. Repertorio de Medicina y Cirugia, 26(1), 9-16. https://doi. org/10.1016/i.reper.2017.04.001

Hoffman, C. (2004). Dumuzi’s Dream: Dream Analysis in Ancient Mesopotamia. Dreaming, 14(4), 240 -251. https://doi.org/10.1037/1053-0797.14.4.240

Idiazabal -Alecha, M. y Fernandez - Prats, M. (2014). Trastornos respiratorios del sueño en la infancia: repercusiones neurocognitivas. Revista de Neurología, 58(1), 83-88.

Jacobson, E. (1938). You can sleep well. McGraw-Hill.

Kabat-Zinn, J. (2007). La practica de la atencion plena. Kairos.

Kales, A. y Kales, J. (1984). Evaluation and treatment of insomnia. Oxford University Press.

Kornum, B., Knudsen, S., Ollila, H., Pizza, F., Jennum, P., Dauvilliers, Y. y Overeem, S. (2017). Narcolepsy. Nature Reviews, 3, Artículo 16100. https://doi.org/10.1038/nrdp.2016.100

Lacks, P., Bertelson, A., Gans, L. y Kunkel, J. (1983). The effectiveness of three behavioral treatments for different degrees of sleep onset insomnia. Behavior Therapy, 14(5), 593 - 605. https://doi.org/10.1016/S00057894(83)80052-5

Lombardo-Aburto, E. (2011). Relación entre trastornos del sueño, rendimiento académico y obesidad en estudiantes de preparatoria. Acta Pediátrica de México, 32(3), 163-168. https://doi.org/10.18233/APM32No3pp163-168

Loomis, A., Harvey, E. y Hobart, G. (1937). Cerebral states during sleep as studied by human brain potentials. Journal Experimental Psychology, 21, 127-144.

Lovato, N., Miller, C., Gordon, C., Grunstein, R. y Lack, L. (2018). The Efficacy of Biofeedback for the Treatment of Insomnia: a critical review. Sleep Medicine, 56, 192-200. https://doi.org/10.1016/j.sleep.2018.12.011

Luyster, F., Buysse, D. y Strollo, P. (2010). Comorbid insomnia and obstructive sleep apnea: challenges for clinical practice and research. Jorunal Clinical. Sleep Medicine, 6(2), 196-204.

Luyster, F., Ritterband, L. M., Sereika, S. M., Buysse, D. J., Wenzel, S. E. y Strollo, P. J. (2020). Internet-Based Cognitive-Behavioral Therapy for Insomnia in Adults With Asthma: A Pilot Study. Behaviour Sleep Medicine, 18(1), 10-22. https://doi.org/10.1080/15402002.2018.1518229

Majid, H. y Hirshkowitz, M. (2010). Therapeutics of narcolepsy. Sleep Medicine Clinical 5(4), 659-673. https://doi.org/10.1016/j.jsmc.2010.08.007

Marín-Agudelo, H. y Vinaccia, S. (2005). Modelo cognitivo comportamental del sindrome de narcolepsia-cataplejia: exposicion teorica. Revista de Psicopatología y Psicología Clínica, 10(3), 153-172. https://doi. org/10.5944/rppc.vol.10.num.3.2005.4000

Marín-Agudelo, H., Jimenez Correa, U., Carlos Sierra, J., Pandi Perumal, S. y Schenck, C. (2014). Cognitive 
behavioral treatment for narcolepsy: ca it complement pharmacotherapy? Sleep Science, 7(1), 30-42. https:// doi.org/10.1016/j.slsci.2014.07.023

Medrano-Martínez, P. y Ramos-Platón, M. (2014). Generación y funciones de los ensueños. Revista de Neurología, 59(8), 359-370. https://doi.org/10.33588/rn.5908.2014237

Medrano-Martínez, P. y Ramos-Platón, M. J. (2016). Aleteraciones cognitivas y emocionales en el insomnio crónico. Revista de Neurología, 62(4), 170-178. https://doi.org/10.33588/rn.6204.2015334

Mignot.,E.(1998). Genetic and familial aspects of narcolepsy. Neurology,50(1), 16-22. https://doi.org/10.1212/ WNL.50.2 Suppl 1.S16

Millet, J. (1938). Insomnia: Its cause and treatment. Guilford.

Moloney, M., Martinez, A., Badour, C. y Moga, D. (2019). Internet-Based Cognitive Behavioral Therapy for Insomnia in Appalachian Women: A Pilot Study. Behaviour Sleep Medicine, 30, 1-10. https://doi.org/10.10 $\underline{80 / 15402002.2019 .1661249}$

Monroe, L. (1967). Psychological and physiological differences between good and poor sleepers. Journal of Abnormal Psychology, 72(3), 255-264. https://doi.org/10.1037/h0024563

Montserrat Canal, J. y Puertas Cuesta, F. (2015). Patología Básica del Sueño. Elsevier.

Morin, C., Bootzin, R., Buysse, D., Edinger, J., Espie, C., y Linchstein, K. (2006). Psychological and behavioral treatment of insomnia: Update of the recent evidence. Sleep, 29(11), 1398-1414. https://doi.org/10.1093/ $\underline{\text { sleep/29.11.1398 }}$

Morin, C. y Benca, R. (2012). Chronic insomnia. Lancet, 379, 1129-1141.

Morin, C. (1993). Insomnia psychological assessment and management. Guilford Press.

Morin, C. (1994). Dysfunctional beliefs and attitudes about sleep: Preliminary scale development and description. The Behavior Therapist, 30(11), 1547-1554. https://doi.org/10.1093/sleep/30.11.1547

Morin, C. (2006). Cognitive-behavioral Therapy of Insomnia. Sleep Medicine Clinics, 1(3), 375-386. https:// doi.org/10.1016/j.jsmc.2006.06.008

Morin, C. y Espie, C. (2003). Insomnia: A Clinical Guide to Assessment and Treatment. Plenum Publishing Corp.

Morin, C., Hauri, P., Espie, C., Spielman, A., Buysse, D. y Bootzin, R. (1999). Nonpharmacologic Treatment of Chronic Insomnia. Sleep, 22(8), 1134-1156. https://doi.org/10.1093/sleep/22.8.1134

Morin, C., Stone, J., Trinkle, D., Mercer, J. y Rembserg, S. (1993). Dysfunctional beliefs and attitudes about sleep among older adults with and without insomnia complaints. Psychology and Aging, 8(3), 463-467. https://doi.org/10.1037/0882-7974.8.3.463

Morin, C., Vallieres, A., Guay, B., Ivers, H., Savard, J., Merette, C., Bastien, C. y Baillargeon, L. (2009). Cognitive behavioral therapy, singly and combined with medication, for persistent insomnia: a randomized controlled trial. Journal of the American Medical Association, 301(19), 2005-2015. https://doi.org/10.1001/ jama.2009.682

Mullington, J. y Broughton, R. (1993). Scheduled naps in the management of daytime sleepiness in narcolepsy-cataplexy. Sleep, 16(5), 444-456. https://doi.org/10.1093/sleep/16.5.444

Nicassio, P. y Bootzin, R. (1974). Comparison of progressive relaxation and autogenic training as treatments for insomnia. Journal of Abnormal Psychology, 83(3), 253-260. https://doi.org/10.1037/h0036729

Ohayon, M. (2011). Epidemiological Overview of Sleep Disorders in the General Population. Sleep Medical Research, 2(1), 1-9. https://doi.org/10.17241/smr.2011.2.1.1

Overeem, S., Reading, P. y Bassetti, C. (2012). Narcolepsy. Sleep Medicine Clinics, 7(2), 263-281. https://doi. org/10.1016/j.jsmc.2012.03.013

Pabon, R., García de Gurtubay, I., Morales, G., Urriza, J., Imirizaldu, L. y Ramos-Argüelles, F. (2010). Narcolepsia: actualización en etiología, manifestaciones clínicas y tratamiento. Anales. Sistema. Sanitario de Navarra, 33(2), 191-201.

Paez-Moya, S. y Parejo-Gallardo, K. (2017). Cuadro clínico del síndrome de apnea-hipopnea. Revista de la Factual de Medicina, 65(1), 29-37. https://doi.org/10.15446/revfacmed.v65n 1Sup.59726

Perlis, M. L., Jungquist, C., Smith, M. T. y Posner, D. (2009). Insomnio. Una guía cognitivo-conductual de tratamiento. Desclee de Brouwer. 
Perlis, M., Shaw, O., Cano, G. y Espie, C. (2011). Models of insomnia. En M. Kyger, T. Roth y W. Dement. Principles and practice of sleep medicine. ( $5^{\mathrm{a}}$ ed., pp. 850-865). Saunders.

Pich, C., Bach, M., Popp, R., Jara, C., Crönlein, T., Hajak, G. y Geisler, P. (2013). Insomnia symptoms influence CPAP compliance. Sleep Breath, 17(1), 99-104. https://doi.org/10.1007/s11325-012-0655-9

Pigeon, W., Funderburk, J., Bishop, T. y Crean, H. (2017). Brief cognitive behavioral therapy for insomnia delivered to depressed veterans receiving primary care services: A pilot study. Journal of Affective Disorders, 217, 105-111. https://doi.org/10.1016/j.jad.2017.04.003

Ram, S., Seirawan, H., Kumar, S. y Clark, G. (2010). Prevalence and impact of sleep disorders and sleep habits in the United States. Sleep Breath, 14(1), 63-70. https://doi.org/10.1007/s11325-009-0281-3

Rash, J., Kavanagh, V. y Garland, S. (2019). A Meta-Analysis of Mindfulness-Based Therapies for Insomnia and Sleep Disturbance: Moving Towards Processes of Change. Sleep Medicine Clinics, 14(2), 209-233. https://doi.org/10.1016/j.jsmc.2019.01.004

Reynoso-Erazo, L. y Becerra-Galvez, A. (2014). Medicina conductual: Teoría y Práctica. Qartuppi S. Riemann, D., Spiegelhalder, K., Feige, B., Voderholzer, U., Berger, M., Perlis, M. y Nissen, C. (2010). The hyperarousal model of insomnia: a review of the concept and its evidence. Sleep Medicine Reviews, 14(1), 19-31. https://doi.org/10.1016/j.smrv.2009.04.002

Rogers., A. y Aldric, M. (1993). The effect of regularly scheduled naps on sleep attacks and excessive daytime sleepiness associated with narcolepsy. Nursery Research, 42(2), 111-117.

School, D. y Foundation, W. E. (n.d.). The Science of Sleep. http://healthysleep.med.harvard.edu/healthy/matters/benefits-of-sleep/why-do-we-sleep

Sivertsen, B., Omvik, S., Palleson, S., Bjorvatn, B., Havik, O. E., Kvale, G., Nielsen, G. H. y Nordhus, I. H. (2006). Cognitive behavioral therapy vs zopiclone for treatment of chronic primary insomnia in older adults; a randomized controlled trial. Journal of the Amercian Medical Association, 295(24), 2851-2858. https://doi. org/10.1001/jama.295.24.2851

Soriano Mas, C. (2007). Fundamentos de neurociencias. Editorial UOC.

Spielman, A., Saskin, P.y Thorpy, M. J. (1987). Treatment of Chronic Insomnia by Restriction of Time in Bed. Sleep, 10(1), 45-56.

Stepanski, E. (2003). Behavioral sleep medicine: A historical perspective. Behavioral sleep medicine, 1(1), 4-21. https://doi.org/10.1207/S15402010BSM0101 3

Sweetman, A., Lack, L., Catcheside, P., Antic, N., Smith, S., Chai-Coetzer, C., Douglas, J., O’Grady, A., Dunn,N., Robinson,J., Paul, D., Williamson,P. y McEvoy, R. (2019). Cognitive and behavioral therapy for insomnia increases the use of continuous positive airway pressure therapy in obstructive sleep apnea participants with comorbid insomnia: a randomized clinical trial. Sleep Research Society, 42(12), 1-12. https://doi. org/10.1093/sleep/zsz178

Sweetman, A., Lack, L., Smith, S., Catcheside, P., Antic , N., Chai-Coetzer , C., Douglas, J., O’Grady, A., Dunn, N., Robinson, J. y McEvoy, D. (2017). Changes in epworth sleepiness scale during bedtime restriction therapy in co-morbid insomnia and obstructive sleep apnea. Sleep, 40(1), 139-140. https://doi.org/10.1093/ sleepj/zsx050.374

Sweetman, A., McEvoy, R., Smith, S., Catcheside, P., Antic, N., Chai-Coetzer, C. C., Douglas, J., O’Grady, A., Dunn, N., Robinson, J., Paul, D., Paul, W. y Lack, L. (2020). The effect of cognitive and behavioral therapy for insomnia on week-to-week changes in sleepiness and sleep parameters in insomnia patients with co-morbid moderate and severe sleep apnea: A randomized controlled trial. Sleep 47(7), Artículo zsaa002. https://doi.org/10.1093/sleep/zsaa002

Tanaka, M., Kusaga, M., Nyamathi, A. y Tanaka, K. (2019). Effects of Brief Cognitive Behavioral Therapy for Insomnia on Improving Depression Among Community-Dwelling Older Adults: A Randomized Controlled Comparative Study. Worldviews Evidence Based Nursing, 16(1), 78-86. https://doi.org/10.1111/wvn.12342

Tena-Razo, A. y Jiménez-Genchi, A. (2017). Efficacy of cognitive behavioral therapy for insomnia in patients with insomnia comorbid with major depression. Sleep, 40(1), 408-409. https://doi.org/10.1093/sleepj/ zSx050.1096

Thorpy, M. (1997). Hystory of Sleep. Medicine Sleep. Multimedia version 2.5. 
Tirapu-Ustárroz, J. (2012). Neuropsicología de los sueños. Revista de Neurología, 55(2), 101-110. https://doi. org/10.33588/rn.5502.2012149

Trauer, J., Qian, M., Doyle, J., Rajaratnam, S. y Cunnington, D. (2015). Cognitive Behavioral Therapy for Chronic Insomnia: A Systematic Review and Meta-analysis. Annals of Internal Medicine, 163(3), 191-204. https://doi.org/10.7326/M14-2841

Van Someren, E. J., Cirelli, C., Dijk, D.-J., Cauter, E. V., Schwartz, S. y Chee, M. W. (2015). Disrupted Sleep: From Molecules to Cognition. The Journal of Neuroscience, 35(41), 13889-13895. https://doi.org/10.1523/ JNEUROSCI.2592-15.2015

Viola, A., Archer, S., James, L., Groeger, J., Lo, J., Skene, D., Von Schantz, M. y Dik, D. (2007). PER3 polymorphism predicts sleep structure and waking performance. Current Biology, 17(7), 613- 618. https://doi. org/10.1016/j.cub.2007.01.073

Weil, G. y Goldfried, M. (1973). Treatment of Insomnia in an Eleven-Year-Old Child Through Self-Relaxation. Behavior Therapy, 4(2), 282-294.

Wickwire, E., Smith, M., Birnbaum, S. y Collop, N. (2010). Sleep maintenance insomnia complaints predict poor CPAP adherence: A clinical case series. Sleep Medicine, 11(8), 772-776. https://doi.org/10.1016/j. sleep.2010.03.012

Wilhem, I., Diekelmann, S., Molzow, I., Ayoub, A., Molle, M. y Born, J. (2011). Sleep selectively enhances memory expected to be of future relevance. The Journal of Neuroscience, 31(5), 1563-1569. https://doi. org/10.1523/JNEUROSCI.3575-10.2011

Wozniak, D., Lasserson, T. y Smith, I. (2009). Educational, supportive and behavioural interventions to improve usage of continuous positive airway pressure machines in adults with obstructive sleep apnoea. Cochrane Database Systematische Reviews, 2, Artículo CD007736. https://doi.org/10.1002/14651858.CD007736

Artículo recibido: 04/03/2020

Artículo aceptado: 13/06/2020 